\title{
DESCRIPTION OF ELASTIC ANISOTROPY OF QUASICRYSTALLINE STRUCTURES USING A DISCRETE ATOMISTIC APPROACH
}

\author{
S. S. Stvolova*, I. Yu. Zubko \\ Perm National Research Polytechnic University, 29, Komsomolski Pr Perm, Russian Federation \\ *Corresponding author. E-mail: sofi1234@mail.ru; zoubko@list.ru; address for correspondence: 29, Komsomolski Pr, \\ 614990, Perm, Russian Federation.
}

Prediction of the physical and mechanical properties of nanostructured materials is generally realized within discrete atomistic simulation. Such approach often provides a unique way of studying nanomaterials and requires some restrictions imposed on the used interatomic potentials. A huge amount of different potentials has been used; namely, pairwise, many-particle potentials, the embedded atom method, covalent bond potentials etc. It is well known that, in some cases, computed mechanical properties may differ from experimental data even qualitatively. The paper aims at the demonstration of the ability of different potentials to explain elastic anisotropy by studying invariant representation of the tensor of elastic moduli in the exact form, which has been built using different potentials of interatomic interaction. This makes it possible to study the abilities of different potentials in order to describe the anisotropy of elastic response. The paper demonstrates the ability of two-particle or multi-particle potentials of interatomic interaction on the basis of the Morse potential for the description of the anisotropy of elastic material properties using the obtained invariant representation with an example of two-dimensional quasi-crystalline structures. The pairwise potentials, in contrast to the many-particle embedded atom potential, are shown to be unable to explain elastic anisotropy.

Keywords: discrete-atomistic approach, elastic anisotropy, plain quasi-crystals, manyparticle potentials, embedded atom method, generalized Morse potential.

DOI: $10.17804 / 2410-9908.2016 .3 .031-041$

\section{References}

1. Arroyo M., Belytschko T. Finite crystal elasticity of carbon nanotubes based on the exponential Cauchy-Born rule. Phys. Rev. B, 2004, vol. 69, iss. 11, p. 5415. DOI: 10.1103/PhysRevB.69.115415.

2. Reddy C.D., Rajendran S., Liew K.M. Equilibrium configuration and continuum elastic properties of finite sized graphene. Nanotechnology, 2006, vol. 17, no. 3, pp. 864-870. DOI: $10.1088 / 0957-4484 / 17 / 3 / 042$.

3. Pozdeev A.A., Trusov P.V., Nyashin Yu.I. Bolshie uprugoplasticheskie deformatsii, teoriya, algoritmy, prilozheniya [Large Elastic-Plastic Deformations, Theory, Algorithms, Applications]. M., Nauka Publ., 1986, 232 p. (In Russian).

4. Clayton J. Nonlinear Mechanics of Crystals. London, Springer, 2011, 715 p.

5. Simonov M.V., Zubko I.Yu. Finding equilibrium lattice parameters of different HCPmonocrystals with use of Mie interatomic potential. Vestnik PNIPU. Mekhanika, 2012, no. 3, pp. 204-217. (In Russian).

6. Zubko I.Yu., Simonov M.V. Energy-based approach to estimation of elastic moduli of finite sized specimens with HCP-lattice. Izvestiya Tomskogo politekhnicheskogo universiteta, 2013, vol. 323, no. 2, pp. 194-200. (In Russian).

7. Zubko I.Yu. Computation of elastic moduli of graphene monolayer in non-symmetric formulation using energy-based approach. Fizicheskaya mezomekhanika, 2015, vol. 18, no. 2, pp. 37-50. (In Russian). 
8. Daw M.S., Baskes M.I. Embedded-atom method: Derivation and application to impurities, surfaces, and other defects in metals. Physical Review B, 1984, vol. 29, no. 12, pp. 6443-6453. DOI: 10.1103/PhysRevB.29.6443.

9. Finnis M.W., Sinclair J.E. A simple empirical N-body potential for transition metals. Philosophical Magazine A, 1984, vol. 50, iss. 1, pp. 45-55. DOI: 10.1080/01418618408244210.

10. Sutton A.P., Chen J. Long-range Finnis-Sinclair potentials. Philosophical Magazine Letters, 1990, vol. 61, iss. 3, pp. 139-146. DOI: 10.1080/09500839008206493.

11. Israilishvili J.N. Intermolecular and surface forces. Academic Press: Harcourt Brace and Company, 1998, 450 pp.

12. Chernykh K.F. Vvedenie v anizotropnuyu uprugost [Introduction into Anisotropic Elasticity]. M., Nauka Publ., 1988, 190 p. (In Russian).

\section{About the authors}

Stvolova Sofya Sergeevna - a 4-th year student of Perm National Research Polytechnic University, Department of Mathematical Modelling of Systems and Processes (e-mail: sofi1234@mail.ru)

Zubko Ivan Yurievitch - Ph.D. in physical and mathematical sciences, Associate Professor, Department of Mathematical Modelling of Systems and Processes, Perm National Research Polytechnic University (e-mail: zoubko@list.ru) 
Подана в журнал: 29.04.2016

УДК 538.911, 539.32

DOI: $10.17804 / 2410-9908.2016 .3 .031-041$

\title{
ОПИСАНИЕ УПРУГОЙ АНИЗОТРОПИИ КВАЗИКРИСТАЛЛИЧЕСКИХ СТРУКТУР С ПОМОЩЬЮ ДИСКРЕТНО-АТОМИСТИЧЕСКОГО ПОДХОДА
}

\author{
С. С. Стволова*, И. Ю. Зубко \\ Пермский национальный исследовательский политехнический университет, \\ Комсомольский пр., 29, Пермь, Российская Федерация \\ *Ответственный автор. Электронная почта: sofi1234@mail.ru; адрес для переписки: 614990, \\ Комсомольский пр., 29, Пермь, Российская Федерация
}

Прогнозирование физико-механических свойств наноструктурированных материалов в рамках дискретно-атомистического моделирования, зачастую представляющего собой единственный способ исследования таких объектов, накладывает ряд требований на используемые потенциалы межатомного взаимодействия. В частности, используемые потенциалы должны описывать анизотропию механических свойств кристаллических материалов. Известно множество потенциалов различного типа - двух- и многочастичные, метод погруженного атома, потенциалы ковалентной связи. Получаемые с их помощью оценки механических свойств в ряде случаев могут даже качественно отличаться от экспериментальных данных. Для демонстрации возможностей различных потенциалов описывать анизотропию упругих свойств кристаллических материалов в работе получено инвариантное представление тензора упругих модулей в виде конечных сумм для потенциалов произвольного типа. Это позволяет исследовать возможности различных потенциалов описывать анизотропию упругого отклика. C помощью полученного инвариантного представления тензора линейноупругих модулей на примере двумерных квазикристаллических структур продемонстрированы возможности нескольких двух- и многочастичных потенциалов, построенных на основе предложенной авторами модификации потенциала Морзе. Показано, что в отличие от многочастичного потенциала погруженного атома, парные потенциалы в принципе не могут описать анизотропии упругих свойств.

Ключевые слова: дискретно-атомистическое моделирование, упругая анизотропия, плоские квазикристаллы, многочастичные потенциалы, метод погруженного атома, обобщеенный потенциал Морзе.

\section{1. Введение}

В связи с широким применением новых армированных наночастицами композиционных и других наноструктурированных материалов возникает необходимость прогнозирования их физико-механических свойств. Классические подходы механики континуума напрямую не применимы к таким объектам, как отдельная наночастица или элемент наноструктурированного материала. Однако тела с малым числом атомов являются удобным объектом для изучения в рамках дискретно-атомистических подходов. В работе рассматриваются материалы с кристаллическим строением с произвольным типом связи, потенциал межатомного взаимодействия для которой записывается в виде, допускающем применение как потенциала погруженного атома для металлической связи, так и многочастичных потенциалов, используемых при описании ковалентной связи в углеродных материалах. Целью работы является исследование возможности потенциалов различного типа описывать анизотропию тензора линейно-упругих свойств.

При постановке задачи оценки упругих свойств наночастиц используются понятия механики сплошной среды, которые к подобным объектам с дискретным строением напря-

Stvolova S.S. et al. / Description of elastic anisotropy quasicrystalline structures using discrete atomistic approach 
мую применять нельзя. Будем считать, что образцу конечного размера ставится в соответствие упругое сплошное тело, проявляющее аналогичную реакцию на приложение внешних механических воздействий. Для определения деформаций кристалла задаются две его конфигурации - начальная и текущая. Однородной деформацией кристаллического нанообразца назовем изменения длин и углов между прямыми линиями, соединяющими атомы, и описываемые однородным тензором второго ранга, который соответствует аффинору $\mathbf{F}$, используемому в механике континуума. Пусть $\mathbf{R}_{k}-$ радиус-вектор произвольного $k$-го атома образца в начальной конфигурации, тогда в текущей конфигурации его положение задается вектором $\mathbf{r}_{k}=\mathbf{F} \cdot \mathbf{R}_{k}$ (правило Коши-Борна $\left.[1,2]\right)$. Задача определения упругих модулей наночастиц рассматривается в энергетической постановке, согласно которой плотность потенциальной энергии деформированного кристаллического образца приравнивается плотности упругой энергии тела и искомые упругие модули находятся как ее вторые производные по параметрам деформирования вблизи отсчетной конфигурации.

При описании малых упругих деформаций твердых тел в большинстве работ используется модель линейно-упругой среды, когда в качестве меры деформаций рассматривается линейный тензор малых деформаций. Закон Гука для анизотропного линейно-упругого тела имеет вид линейной связи тензора напряжений Коши $\boldsymbol{\sigma}$ и тензора $\boldsymbol{\varepsilon}$, задаваемой с помощью анизотропного тензора четвертого ранга П линейно-упругих свойств материала:

$$
\sigma=\Pi: \varepsilon
$$

При использовании симметричных б и $\boldsymbol{\varepsilon}$ тензор П симметричен относительно перестановок внутри первой и последней пар индексов:

$$
\Pi_{i j k l}=\Pi_{j i k l}=\Pi_{i j l k} .
$$

Массовая плотность внутренней энергии $u$ совпадает при отсутствии тепловых явлений с плотностью упругой энергии и для линейно-упругой среды имеет вид:

$$
\hat{\rho} u=\frac{1}{2} \sigma: \varepsilon=\frac{1}{2} \boldsymbol{\varepsilon}: \boldsymbol{\Pi}: \boldsymbol{\varepsilon} .
$$

Эта величина представляет собой положительно определенную квадратичную форму, т.е. $u>0$ при $\boldsymbol{\varepsilon} \neq \mathbf{0}$ и $u=0$ при $\boldsymbol{\varepsilon}=\mathbf{0}$. Отсюда следует дополнительная симметрия П относительно перестановки пар индексов:

$$
\Pi_{i j k l}=\Pi_{k l j i}
$$

Компоненты тензора П (упругие модули) в анизотропном случае имеют ясный физический смысл в материальных осях, связанных со структурой материала, например в кристаллографических осях для монокристаллов или в осях, связанных с ориентацией армирующих элементов композиционного материала. Использование тензора малых деформаций подразумевает при замене системы отсчета допустимость наложения только малого поворота системы отсчета, поэтому закон (1) применим лишь для деформируемых тел, не испытывающих (больших) поворотов относительно инерциальной системы отсчета, в которой решается задача. По отношению к малым поворотам тензор $\varepsilon$ является инвариантным. Второй тензор напряжений Пиолы-Кирхгофа связан с тензором напряжений Коши как $\boldsymbol{\sigma}=J^{-1} \mathbf{F} \cdot \mathbf{P}_{\Pi} \cdot \mathbf{F}^{\mathrm{T}}$ [3] и при малых упругих искажениях выражается через линейный тензор деформаций $\boldsymbol{\varepsilon}=\left(\mathbf{F}+\mathbf{F}^{\mathrm{T}}\right) / 2-\mathrm{I}$ :

$$
\mathbf{P}_{\mathrm{II}}=\mathbf{H}: \boldsymbol{\varepsilon}
$$

Stvolova S.S. et al. / Description of elastic anisotropy quasicrystalline structures using discrete atomistic approach 


\section{2. Энергетический подход при дискретно-атомистическом моделировании}

В общем случае потенциальная энергия системы взаимодействующих атомов [4] представляется как сумма

$$
\Phi=\sum_{i=1}^{M} \Phi_{1}\left(\mathbf{R}_{(i)}\right)+\sum_{i=1}^{M-1} \sum_{j=i+1}^{M} \Phi_{2}\left(\mathbf{R}_{(j i)}\right)+\sum_{i=1}^{M-2} \sum_{j=i+1}^{M-1} \sum_{k=j+1}^{M} \Phi_{3}\left(\mathbf{R}_{(j i)}, \mathbf{R}_{(k i)}, \mathbf{R}_{(k j)}\right)+\ldots
$$

где $\mathbf{R}_{(i)}$ - радиус-вектор, задающий положение $i$-го атома; $\mathbf{R}_{(i j)}=\mathbf{R}_{(j)}-\mathbf{R}_{(i)} ; M-$ полное число атомов образца; $\Phi_{1}\left(\mathbf{R}_{(i)}\right)$ - часть потенциальной энергии атомов, которая не зависит от их взаимодействия, а определяется полем некоторой внешней силы; $\Phi_{2}\left(\mathbf{R}_{(i j)}\right)$ - потенциальная энергия парного взаимодействия или двухчастичный потенциал; $\Phi_{3}\left(\mathbf{R}_{(i j)}, \mathbf{R}_{(k i)}, \mathbf{R}_{(k j)}\right)$ - трехчастичный потенциал. Двухчастичный потенциал межатомного взаимодействия характеризует изменение потенциальной энергии при изменении расстояния между парами атомов. Этот потенциал с помощью некоторой функциональной зависимости описывает, что при сближении два атома начинают отталкиваться, а при удалении притягиваться. Поскольку атомы нельзя сдвинуть бесконечно близко, то в ноле такая функция стремится к бесконечности. При увеличении расстояния между парой атомов эта функция выходит на горизонтальную асимптоту, а сила взаимодействия, модуль которой равен тангенсу угла наклона касательной к графику функции $\Phi_{2}\left(\mathbf{R}_{(i j)}\right)$ стремится к нулю. Трехчастичный потенциал учитывает не только расстояние между двумя атомами, как в случае двухчастичного потенциала, но влияние конфигурации ближайших атомов. Заметим, что силы взаимодействия каждого выбранного атома со всеми остальными атомами образца, вычисляемые с помощью двухчастичного потенциала, аддитивны. Для многочастичных потенциалов аддитивности сил взаимодействия нет. В рассматриваемом случае действие внешних сил не рассматривается, поэтому далее $\Phi_{1}\left(\mathbf{R}_{(i)}\right)=0$.

При исследовании механических свойств кристаллов рассматриваются конфигурации с однородным распределением атомов, каждая из которых характеризуется набором параметров межатомного расстояния (для простых решеток это один параметр, для сложных решеток параметров может быть несколько). Принимается, что отсчетная конфигурация является равновесной и может быть определена из условия минимума потенциальной энергии набора взаимодействующих атомов по параметрам решетки.

Для расчета упругих модулей кристаллических систем в рамках энергетического подхода в статической постановке [5-7] принимается, что плотность упругой энергии и плотность потенциальной системы взаимодействующих атомов кристалла в текущей конфигурации совпадают. Тогда производные от плотности потенциальной энергии дискретной системы атомов по мерам деформации дадут выражения для вычисления компонент тензоров напряжений (с помощью первых производных) и компонент тензора линейно-упругих свойств (вторые производные). Пусть $\hat{\Phi}(\mathbf{F})$ - полная потенциальная энергия однородно деформированного кристаллического образца в текущей конфигурации. Энергия образца в отсчетной конфигурации $\Phi_{\mathrm{o}}=\hat{\Phi}(\mathbf{I})$ соответствует материалу с заданной кристаллической структурой и минимальным значением потенциальной энергии по параметрам решетки. Относя изменение полной потенциальной энергии деформированного кристаллического образца к его объему в отсчетной конфигурации $\Omega_{\mathrm{o}}$, получим плотность упругой энергии и тензор напряжений Пиолы-Кирхгофа:

$$
\mathbf{P}=\Omega_{\mathrm{o}}^{-1} \partial\left(\hat{\Phi}(\mathbf{F})-\Phi_{\mathrm{o}}\right) / \partial \mathbf{F}^{\mathrm{T}}=\Omega_{\mathrm{o}}^{-1} \partial \hat{\Phi}(\mathbf{F}) / \partial \mathbf{F}^{\mathrm{T}}
$$


Тензор напряжений Коши $\sigma$ находится как $\boldsymbol{\sigma}=J^{1} \mathbf{F} \cdot \mathrm{P}$, где $J=\operatorname{det} \mathbf{F}=\hat{\Omega} / \Omega_{\mathrm{o}}$, т.е. тензор напряжений Коши $\boldsymbol{\sigma}=\hat{\Omega}^{-1} \mathbf{F} \cdot \partial \hat{\Phi}(\mathbf{F}) / \partial \mathbf{F}^{\mathrm{T}}$.

Рассмотрим представление потенциальной энергии в случае учета только двухчастичного взаимодействия, используя для простоты вместо набора векторов, соединяющих различные пары атомов, обозначение $\Delta \mathbf{R}$ и $\Delta \mathbf{r}$ в двух конфигурациях:

$$
\hat{\Phi}(\mathbf{F})=\sum \hat{\Phi}(|\Delta \mathbf{r}|)=\sum \hat{\Phi}(|\mathbf{F} \cdot \Delta \mathbf{R}|)=\sum \hat{\Phi}\left(\sqrt{\Delta \mathbf{R} \cdot \mathbf{F}^{\mathrm{T}} \cdot \mathbf{F} \cdot \Delta \mathbf{R}}\right)
$$

где $\sum(\cdot)$ - упрощенное обозначение суммы потенциалов (6). Производная по тензору деформационного градиента $\mathbf{F}$ в этом случае приводит к выражению:

$$
\Omega_{\mathrm{o}} \mathbf{P}=\partial \hat{\Phi}(\mathbf{F}) / \partial \mathbf{F}^{\mathrm{T}}=\sum \hat{\Phi}^{\prime}(|\Delta \mathbf{r}|) \partial \sqrt{\Delta \mathbf{R} \cdot \mathbf{F}^{\mathrm{T}} \cdot \mathbf{F} \cdot \Delta \mathbf{R}} / \partial \mathbf{F}^{\mathrm{T}}=\sum \hat{\Phi}^{\prime}(|\Delta \mathbf{r}|)|\Delta \mathbf{r}|^{-1} \Delta \mathbf{R} \Delta \mathbf{r}
$$

следовательно, для тензора напряжений Коши получим выражение

$$
\boldsymbol{\sigma}=\hat{\Omega}^{-1} \sum \hat{\Phi}^{\prime}(|\Delta \mathbf{r}|) \Delta \mathbf{r} \Delta \mathbf{r} /|\Delta \mathbf{r}|, \mathbf{P}_{\mathrm{II}}=\Omega_{\mathrm{o}}^{-1} \sum \hat{\Phi}^{\prime}(|\Delta \mathbf{r}|) \Delta \mathbf{R} \Delta \mathbf{R} /|\Delta \mathbf{r}|,
$$

симметричное для любого потенциала межатомного взаимодействия.

При учете многочастичного взаимодействия в методе погруженного атома [8-10] потенциальная энергия системы атомов определяется выражением, в котором учтено, что отталкивание всех атомов описывается согласно закону парного взаимодействия, а притяжение описывается нелинейной функцией $\gamma($.$) , задающей влияние окружения произвольного атома$ (функция погружения). Окружение определяется множеством $S_{i}$ номеров атомов, участвующих в формировании электронного газа вокруг $i$-го атома:

$$
\hat{\Phi}(\mathbf{F})=\sum_{i=1}^{M-1} \sum_{j=i+1}^{M} \varphi^{+}\left(\mid \mathbf{r}_{(j i)}\right)+\sum_{i=1}^{M} \gamma\left(\sum_{j \in S_{i}} \varphi^{-}\left(\mathbf{r}_{(j i)}\right)\right) .
$$

Производная от первого слагаемого даст выражение, аналогичное полученной ранее сумме. Второе слагаемое - при использовании обозначений $\left(\varphi^{-}\left(\mathbf{r}_{(i j)}\right)\right)^{\prime} \equiv \mathbf{f}_{(i j)}^{-}$:

$$
\begin{gathered}
d \gamma\left(\sum_{j \in S_{i}} \varphi^{-}\left(\mathbf{r}_{(i j)}\right)\right) / d \mathbf{F}^{\mathrm{T}}=\gamma^{\prime}\left(\sum_{j \in S_{i}} \varphi^{-}\left(\mathbf{r}_{(i j)}\right)\right) \sum_{j \in S_{i}} \mathbf{R}_{(i j)} \mathbf{f}_{(i j)}^{-} ; \\
\mathbf{P}=\Omega_{\mathrm{o}}^{-1}\left\{\sum_{i=1}^{M-1} \sum_{j=i+1}^{M}\left(\varphi^{+}\right)^{\prime}\left|\mathbf{r}_{(i j)}\right|^{-1} \mathbf{R}_{(i j)} \mathbf{r}_{(i j)}+\sum_{i=1}^{M}\left(\gamma^{\prime} \sum_{j \in S_{i}} \mathbf{R}_{(i j)} \mathbf{f}_{(i j)}^{-}\right)\right\} ; \\
\boldsymbol{\sigma}=\hat{\Omega}^{-1}\left\{\sum_{i=1}^{M-1} \sum_{j=i+1}^{M}\left(\varphi^{+}\right)^{\prime}\left|\mathbf{r}_{(i j)}\right|^{-1} \mathbf{r}_{(i j)} \mathbf{r}_{(i j)}+\sum_{i=1}^{M}\left(\gamma^{\prime} \sum_{j \in S_{i}} \mathbf{r}_{(i j)} \mathbf{f}_{(i j)}^{-}\right)\right\} ; \\
\mathbf{P}_{\mathrm{II}}=\Omega_{\mathrm{o}}^{-1}\left\{\sum_{i=1}^{M-1} \sum_{j=i+1}^{M}\left(\varphi^{+}\right)^{\prime}\left|\mathbf{r}_{(i j)}\right|^{-1} \mathbf{R}_{(i j)} \mathbf{R}_{(i j)}+\sum_{i=1}^{M}\left(\gamma^{\prime} \sum_{j \in S_{i}} \mathbf{R}_{(i j)} \mathbf{F}_{(i j)}^{-}\right)\right\},
\end{gathered}
$$

где $\mathbf{F}_{(i j)}^{-} \equiv \mathbf{F}^{-1} \cdot \mathbf{f}_{(i j)}^{-}$. Так как вектор $\mathbf{f}_{(i j)}^{-}$(или $\mathbf{F}_{(i j)}^{-}$) не обязательно направлен вдоль $\mathbf{r}_{(i j)}$ (или $\left.\mathbf{R}_{(i j)}\right)$, то получаемые тензоры (10-11) могут быть несимметричными.

Для вычисления упругих модулей в несимметричном случае при малых деформациях необходимо найти производную от тензора напряжений (11): 


$$
d^{2} u / d \mathbf{F}^{2}=\Omega_{\mathrm{o}}^{-1} d\left(\sum_{i=1}^{M-1} \sum_{j=i+1}^{M}\left(\varphi^{+}\right)^{\prime}\left|\mathbf{r}_{(i j)}\right|^{-1} \mathbf{r}_{(i j)} \mathbf{R}_{(i j)}+\sum_{i=1}^{M}\left(\gamma^{\prime} \sum_{j \in S_{i}} \mathbf{f}_{(i j)}^{-} \mathbf{R}_{(i j)}\right)\right) / d \mathbf{F} .
$$

В итоге получим тензор четвертого ранга:

$$
\begin{aligned}
& \Omega_{\mathrm{o}} \partial \mathbf{P}_{\mathrm{II}} / \partial \mathbf{F}=\sum_{1 \leq i<j \leq M}\left\{\frac{\left(\varphi^{+}\right)^{\prime \prime}\left|\mathbf{r}_{(i j)}\right|-\left(\varphi^{+}\right)^{\prime}}{\left|\mathbf{r}_{(i j)}\right|^{3}} \mathbf{r}_{(i j)} \mathbf{R}_{(i j)} \mathbf{r}_{(i j)} \mathbf{R}_{(i j)}+\frac{\left(\varphi^{+}\right)^{\prime}}{\left|\mathbf{r}_{(i j)}\right|} \mathbf{e}_{k} \mathbf{R}_{(i j)} \mathbf{e}^{k} \mathbf{R}_{(i j)}\right\}+ \\
& +\sum_{i=1}^{M}\left\{\gamma^{\prime \prime}\left(\sum_{j \in S_{i}} \mathbf{R}_{(i j)} \mathbf{f}_{(i j)}^{-}\right)\left(\sum_{j \in S_{i}} \mathbf{R}_{(i j)} \mathbf{f}_{(i j)}^{-}\right)+\gamma^{\prime} \sum_{j \in S_{i}}\left(\mathbf{g}_{(i j)} \cdot \mathbf{e}_{k} \mathbf{R}_{(i j)} \mathbf{e}^{k} \mathbf{R}_{(i j)}\right)\right\},
\end{aligned}
$$

где $\mathbf{e}_{k}$ - векторы фиксированного пространственного базиса; производные $\gamma^{\prime}$ и $\gamma^{\prime \prime}$ функции $\gamma$ имеют аргумент $\sum_{j \in S_{i}} \varphi^{-}\left(\mathbf{r}_{(i j)}\right)$, а тензор второго ранга $\mathbf{g}_{(i j)}$ определен как $\mathbf{g}_{(i j)} \equiv$ $\partial \mathbf{f}_{(i j)}^{-} / \partial \mathbf{r}_{(i j)}=\partial^{2} \varphi^{-}\left(\mathbf{r}_{(i j)}\right) / \partial \mathbf{r}_{(i j)}{ }^{2}$. При малых деформациях $\mathbf{F} \rightarrow \mathbf{I}$ инвариантное представление тензора Н линейно-упругих модулей для кристаллического материала в отсчетной конфигурации имеет вид:

$$
\begin{gathered}
\Omega_{\mathrm{o}} \mathbf{H}=\sum_{1 \leq i<j \leq M}\left\{\frac{\left(\varphi^{+}\right)^{\prime \prime}\left|\mathbf{R}_{(i j)}\right|-\left(\varphi^{+}\right)^{\prime}}{\left|\mathbf{R}_{(i j)}\right|^{3}} \mathbf{R}_{(i j)} \mathbf{R}_{(i j)} \mathbf{R}_{(i j)} \mathbf{R}_{(i j)}+\frac{\left(\varphi^{+}\right)^{\prime}}{\left|\mathbf{R}_{(i j)}\right|} \mathbf{e}_{k} \mathbf{R}_{(i j)} \mathbf{e}^{k} \mathbf{R}_{(i j)}\right\}+ \\
+\sum_{i=1}^{M} \sum_{j \in S_{i}}\left\{\gamma^{\prime \prime}\left(\sum_{j \in S_{i}} \mathbf{R}_{(i j)} \mathbf{F}_{(i j)}^{-}\right)\left(\sum_{j \in S_{i}} \mathbf{R}_{(i j)} \mathbf{F}_{(i j)}^{-}\right)+\gamma^{\prime} \sum_{j \in S_{i}}\left(\mathbf{G}_{(i j)} \cdot \mathbf{e}_{k} \mathbf{R}_{(i j)} \mathbf{e}^{k} \mathbf{R}_{(i j)}\right)\right\},
\end{gathered}
$$

где $\mathbf{G}_{(i j)}=\partial^{2} \varphi^{-}\left(\mathbf{R}_{(i j)}\right) / \partial \mathbf{R}_{(i j)}{ }^{2} ; \mathbf{F}_{(i j)}^{-}=d \varphi^{-}\left(\mathbf{R}_{(i j)}\right) / d \mathbf{R}_{(i j)}$. В общем случае выражение (12) не обладает симметрией в парах первых и вторых диад, которая возможна только в случае $\mathbf{F}_{(i j)}^{-} \| \mathbf{R}_{(i j)}$. Без учета погружения $\gamma(x)=-1 / 2 x$ :

$$
\begin{gathered}
\Omega_{\mathrm{o}} \mathbf{H}=\sum_{1 \leq i<j \leq M}\left\{\frac{\left(\varphi^{+}\right)^{\prime \prime}\left|\mathbf{R}_{(i j)}\right|-\left(\varphi^{+}\right)^{\prime}}{\left|\mathbf{R}_{(i j)}\right|^{3}} \mathbf{R}_{(i j)} \mathbf{R}_{(i j)} \mathbf{R}_{(i j)} \mathbf{R}_{(i j)}+\frac{\left(\varphi^{+}\right)^{\prime}}{\left|\mathbf{R}_{(i j)}\right|} \mathbf{e}_{k} \mathbf{R}_{(i j)} \mathbf{e}^{k} \mathbf{R}_{(i j)}\right\}- \\
-\sum_{1 \leq i<j \leq M}\left\{\mathbf{G}_{(i j)} \cdot \mathbf{e}_{k} \mathbf{R}_{(i j)} \mathbf{e}^{k} \mathbf{R}_{(i j)}\right\} .
\end{gathered}
$$

\section{3. Исследование упругой анизотропии для различных потенциалов}

Рассмотрим применение полученных соотношений в частном случае двумерных квазикристаллических структур. Для описания металлической связи в дискретноатомистическом моделировании используется метод погруженного атома [8-10], являющийся примером многочастичного потенциала. Для того чтобы группы атомов взаимодействовали на большом расстоянии согласно экспериментальным законам (описываются потенциалами Морзе или Ми [11]), а на малых расстояниях учитывалась металлическая (многочастичная) связь, предлагается модификация метода, основанная на применении обобщенного потенциала Морзе: 


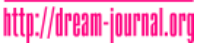

$$
\begin{gathered}
\frac{\Phi}{\beta}=\frac{1}{m-n}\left[\sum_{i=1}^{M-1} \sum_{j=i+1}^{M}\left\{n \exp \left(m \gamma\left(\alpha-\left|\mathbf{r}_{(j i)}\right|\right)\right)-m\left(1-c_{i j}\right) \exp \left(n \gamma\left(\alpha-\left|\mathbf{r}_{(j i)}\right|\right)\right)\right\}-\right. \\
\left.-\frac{m}{2} \sum_{i=1}^{M}\left\{\sum_{j=1, j \neq i}^{M} c_{i j} \exp \left(n \gamma\left(\left|\mathbf{r}_{(j i)}\right|-\alpha\right)\right)^{p}\right\}^{1 / p}\right],
\end{gathered}
$$

где $M$ - число всех атомов образца; $m, n \in \mathbb{Z} ; c_{i j} \in\{0,1\} ; p \in\{1,2\} ; c_{i j}=1$ для атомов, участвующих в образовании электронного газа вблизи положения $i$-го атома; $c_{i j}=0$ для всех остальных атомов. Размер окрестности для учета соседей может быть от одной до нескольких координационных сфер. Также заданием параметров $c_{i j}$, учитывающих для каждого атома взаимное притяжение к нему трех ближайших соседей, может быть описана и ковалентная связь [7]. При $p=1$ и любом числе атомов или для двух изолированных атомов $M=2$ и любого $p$ потенциал (13) совпадает с обобщенным потенциалом Морзе:

$$
\frac{\Phi}{\beta}=\frac{1}{m-n} \sum_{i=1}^{M-1} \sum_{j=i+1}^{M}\left\{n \exp \left(m \gamma\left(\alpha-\left|\mathbf{r}_{(j i)}\right|\right)\right)-m \exp \left(n \gamma\left(\alpha-\left|\mathbf{r}_{(j i)}\right|\right)\right)\right\}
$$

При условии, что для всех атомов $c_{i j}=0$ также получается обобщенный потенциал Морзе, при $p=2$ получается аналог потенциала Финниса-Синклэра [9]. Параметр $\alpha$ задает равновесное расстояние для изолированной пары атомов; $\beta$ - энергия связи этих двух атомов. При $m=2$ и $n=1$ получается классический потенциал Морзе:

$$
\frac{\Phi}{\beta}=\frac{1}{m-n} \sum_{i=1}^{M-1} \sum_{j=i+1}^{M}\left\{\exp \left(-2 \gamma\left(\left|\mathbf{r}_{(j i)}\right|-\alpha\right)\right)-2 \exp \left(-\gamma\left(\left|\mathbf{r}_{(j i)}\right|-\alpha\right)\right)\right\}
$$

Для плоских квазикристаллических структур с осями симметрии различного порядка (рисунок) получено, что при использовании парных потенциалов для оси симметрии любого порядка, кроме четвертого, тензор упругих свойств содержит только две ненулевые независимые компоненты. При этом коэффициент Пуассона для этих структур равен 1/3, т.е. в действительности получался только один независимый упругий модуль (таблица). Для кристалла с осью симметрии 4-го порядка коэффициент Пуассона отрицателен и существует два независимых упругих модуля. Во всех случаях начальная равновесная конфигурация определялась из условия минимума полной потенциальной энергии системы по параметру $a$ межатомного расстояния (рисунок). Объемная плотность упругой энергии структур определялась по отношению к площади образцов. Известная из экспериментов и теоретических оценок зависимость механических свойств от размеров тела наблюдалась и для рассмотренных структур, хотя отдельно этот вопрос не исследовался, поскольку целью была проверка способности различных потенциалов описывать анизотропию упругих свойств, характер которой не связан с размерами образца. 


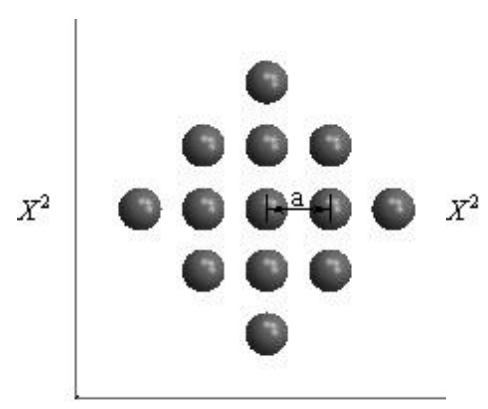

$X^{1}$

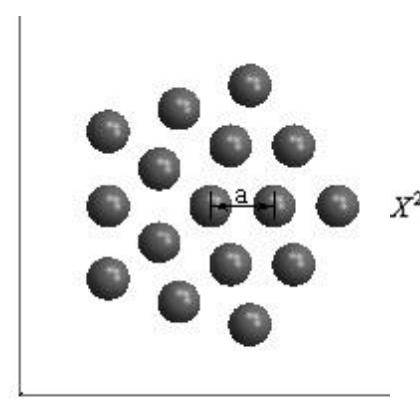

$X^{1}$

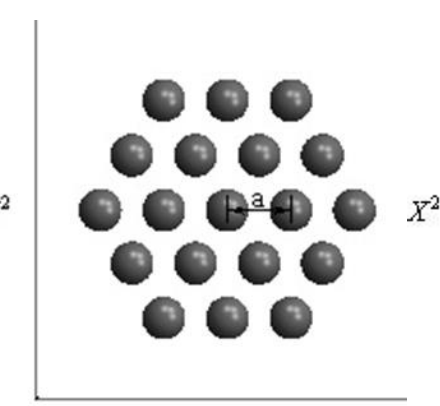

$X^{1}$

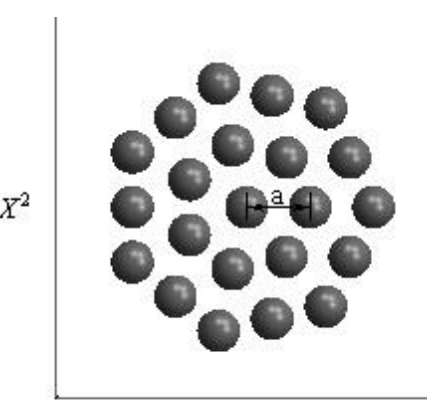

$X^{1}$

$\sigma$

B

Элементы плоских квазикристаллических структур с осями симметрии: $a$ - 4-го порядка; $\sigma$ - 5-го порядка; в - 6-го порядка; 2 - 7-го порядка

Значения упругих модулей, коэффициента Пуассона и модуля Юнга при различных потенциалах межатомного взаимодействия и коэффициентах $\mathrm{m}$ и $\mathrm{n}$

\begin{tabular}{|l|l|l|l|l|}
\hline \multirow{2}{*}{ Потенциал } & \multicolumn{4}{|c|}{ Ось симетрии } \\
\cline { 2 - 5 } & 4-го порядка & 5-го порядка & 6-го порядка & 7-го порядка \\
\hline Потенциал & $H_{1111}=H_{2222}=$ & $H_{111}=H_{2222}=$ & $H_{1111}=H_{2222}=$ & $H_{111}=H_{2222}=$ \\
Морзе & $=25.52 \beta / \alpha^{2}$ & $=22.78 \beta / \alpha^{2}$ & $=24.48 \beta / \alpha^{2}$ & $=22.41 \beta / \alpha^{2}$ \\
$m=2, n=1$, & $H_{1122}=H_{1212}=$ & $H_{1122}=H_{1212}=$ & $H_{1122}=H_{1212}=$ & $H_{1122}=H_{1212}=$ \\
$\alpha \gamma=3.14$ & $=-2.63 \beta / \alpha^{2}$ & $=7.59 \beta / \alpha^{2}$ & $=8.16 \beta / \alpha^{2}$ & $=7.47 \beta / \alpha^{2}$ \\
& $E=27.27 \beta / \alpha^{2}$ & $E=20.25 \beta / \alpha^{2}$ & $E=21.76 \beta / \alpha^{2}$ & $E=19.92 \beta / \alpha^{2}$ \\
& $v=-0.09$ & $v=1 / 3$ & $v=1 / 3$ & $v=1 / 3$ \\
Потенциал & $=131.0 \beta / \alpha^{2}$ & $=125.9 \beta / \alpha^{2}$ & $=191.3 \beta / \alpha^{2}$ & $=111.8 \beta / \alpha^{2}$ \\
Морзе & $H_{1122}=H_{1212}=$ & $H_{1122}=H_{1212}=$ & $H_{1122}=H_{1212}=$ & $H_{1122}=H_{1212}=$ \\
$m=5, n=4$, & $=-2.00 \beta / \alpha^{2}$ & $=41.98 \beta / \alpha^{2}$ & $=63.77 \beta / \alpha^{2}$ & $=37.26 \beta / \alpha^{2}$ \\
$\alpha \gamma=3.14$ & $E=131.0 \beta / \alpha^{2}$ & $E=111.9 \beta / \alpha^{2}$ & $E=170.1 \beta / \alpha^{2}$ & $E=99.35 \beta / \alpha^{2}$ \\
& $v=-0.01$ & $v=1 / 3$ & $v=1 / 3$ & $v=1 / 3$ \\
\hline Потенциал & $H_{1111}=H_{2222}=$ & $H_{1111}=H_{2222}=$ & $H_{1111}=H_{2222}=$ & $H_{1111}=H_{2222}=$ \\
$(13)$ & $=2.95 \beta / \alpha^{2}$ & $=2.77 \beta / \alpha^{2}$ & $=2.86 \beta / \alpha^{2}$ & $=2.80 \beta / \alpha^{2}$ \\
$m=2, n=1$, & $H_{1122}=2.03 \beta / \alpha$ & $H_{1122}=2.63 \beta / \alpha^{2}$ & $H_{1122}=2.81 \beta / \alpha^{2}$ & $H_{1122}=2.42 \beta / \alpha^{2}$ \\
$\alpha \gamma=3.14$, & $H_{1212}=-0.06 \beta / \alpha_{1212}=0.07 \beta / \alpha^{2}$ & $H_{1212}=0.03 \beta / \alpha^{2}$ & $H_{1212}=0.19 \beta / \alpha^{2}$ \\
$p=2, c_{i j}=1$ & $E=1.55 \beta / \alpha^{2}$ & $E=0.27 \beta / \alpha^{2}$ & $E=0.10 \beta / \alpha^{2}$ & $E=0.71 \beta / \alpha^{2}$ \\
& $v=0.69$ & $v=0.95$ & $v=0.98$ & $v=0.86$ \\
\hline
\end{tabular}

Из условия положительной определенности тензора линейно-упругих свойств $\mathbf{H}$ для двумерной среды, как в рассмотренных примерах, следуют ограничения $E>0, v \in(-1 ; 1)$. Модуль Юнга определяется соотношением $E=\left(H_{1111}^{2}-H_{1122}^{2}\right) / H_{1111}$; коэффициент Пуассона $-v=H_{1122} / H_{1111}$. При использовании потенциала (13) погруженного атома для различ- 
ных двумерных структур вне зависимости от значений параметров потенциал получалось одинаковое число независимых модулей. Это число лишь частично соответствует результатам линейной теории упругости [12]. Отличие от классических результатов для двумерной среды дает структура с осью симметрии 6-го порядка, которая должна описываться только двумя независимыми ненулевыми упругими модулями. Метод погруженного атома при этом дает три независимых компоненты тензора упругих свойств. Также для всех рассмотренных случаев при использовании потенциала (13) получается заниженное значение сдвигового модуля $G=H_{1212}$. Эти особенности требуют дополнительного исследования. Тем не менее для более сложных случаев симметрии метод погруженного атома, в отличие от парных потенциалов, дает физически более корректные результаты.

\section{4. Заключение}

Для тензора упругих модулей, вычисляемого в статическом подходе при дискретноатомистическом моделировании, получена инвариантная запись. С помощью этого представления в работе показано, что парные потенциалы позволяют описать только изотропию упругих свойств, поэтому их применение для кристаллических тел не всегда допустимо. На примере предложенной авторами статьи модификации метода погруженного атома и обобщенного потенциала Морзе показано, что многочастичные потенциалы позволяют более адекватно прогнозировать упругую анизотропию кристаллов.

\section{Благодарность}

Работа выполнена в рамках задания № 2014/152 на выполнение государственных работ в сфере научной деятельности в рамках базовой части госзадания Минобрнауки РФ (код проекта - 1911), а также при финансовой поддержке Российского фонда фундаментальных исследований (грант №14-01-00069, грант №15-01-08678).

\section{Литература}

1. Arroyo M., Belytschko T. Finite crystal elasticity of carbon nanotubes based on the exponential Cauchy-Born rule // Phys. Rev. B. - 2004. - Vol. 69, iss. 11. - P. 5415. DOI: 10.1103/PhysRevB.69.115415.

2. Reddy C. D., Rajendran S., Liew K. M. Equilibrium configuration and continuum elastic properties of finite sized graphene // Nanotechnology. - 2006. - Vol. 17, no. 3. - P. 864-870. DOI: $10.1088 / 0957-4484 / 17 / 3 / 042$.

3. Поздеев А. А., Трусов П. В., Няшин Ю. И. Большие упругопластические деформации: теория, алгоритмы, приложения. - М. : Наука, 1986. - 232 с.

4. Clayton J. Nonlinear Mechanics of Crystals. - London : Springer, 2011 - 715 p.

5. Симонов М. В., Зубко И. Ю. Определение равновесных параметров решетки различных ГПУ-монокристаллов с помощью потенциала межатомного взаимодействия Ми // Вестник Пермского национального исследовательского политехнического университета. Механика. - 2012. - № 3. - С. 204-217.

6. Зубко И. Ю., Симонов М. В. Энергетический способ расчета упругих модулей образцов конечных размеров с ГПУ-решеткой // Известия Томского политехнического университета. - 2013. - Т. 323, № 2. - С. 194-200.

7. Зубко И. Ю. Вычисление упругих модулей монослоя графена в несимметричной постановке с помощью энергетического подхода // Физическая мезомеханика. - 2015. - Т. 18, № 2. - C. 37-50.

8. Daw M. S., Baskes M. I. Embedded-atom method: Derivation and application to impurities, surfaces, and other defects in metals // Physical Review B. - 1984. - Vol. 29, no. 12. P. 6443-6453. - DOI: 10.1103/PhysRevB.29.6443. 
9. Finnis M. W., Sinclair J. E. A simple empirical N-body potential for transition metals // Philosophical Magazine A. - 1984. - Vol. 50, iss. 1. - P. 45-55. DOI: $10.1080 / 01418618408244210$.

10. Sutton A. P., Chen J. Long-range Finnis-Sinclair potentials // Philosophical Magazine Letters. - 1990. - Vol. 61, iss. 3. - P. 139-146. - DOI: 10.1080/09500839008206493.

11. Israilishvili J. N. Intermolecular and surface forces. - Academic Press: Harcourt Brace and Company, 1998. -450 pp.

12. Черных К. Ф. Введение в анизотропную упругость. - М. : Наука, 1988. - 190 с.

\section{Об авторах}

Стволова Софья Сергеевна - студентка 4-го курса Пермского национального исследовательского политехнического университета, кафедра математического моделирования систем и процессов (e-mail: sofi1234@mail.ru).

Зубко Иван Юрьевич - кандидат физико-математических наук, доцент, кафедра математического моделирования систем и процессов Пермского национального исследовательского политехнического университета (e-mail: zoubko@ list.ru). 